\title{
Localization of P300 Sources in Schizophrenia Patients Using Constrained BSS
}

\author{
Saeid Sanei ${ }^{1}$, Loukianos Spyrou ${ }^{1}$, Wenwu Wang ${ }^{2}$, and Jonathon A. Chambers ${ }^{2}$ \\ ${ }^{1}$ Centre for Digital Signal Processing Research, King's College London, WC2R 2LS, UK \\ saeid.sanei@kcl.ac.uk \\ ${ }^{2}$ Communications and Information Technologies Research Group \\ Cardiff School of Engineering, Cardiff University, Cardiff, CF24 0YF, UK
}

\begin{abstract}
A robust constrained blind source separation (CBSS) algorithm has been proposed for separation and localization of the P300 sources in schizophrenia patients. The algorithm is an extension of the Infomax algorithm, based on minimization of mutual information, for which a reference P300 signal is used as a constraint. The reference signal forces the unmixing matrix to separate the sources of both auditory and visual P300 resulted from the corresponding stimulations. The constrained problem is then converted to an unconstrained problem by means of a set of nonlinear penalty functions. This leads to the modification of the overall cost function, based on the natural gradient algorithm (NGA). The P300 sources are then localized based on electrode - source correlations.
\end{abstract}

\section{Introduction}

Based on clinical investigations, P300 is a positive event-related potential (ERP), which occurs with a latency of about $300 \mathrm{~ms}$ after rare or task relevant stimuli [1]. This is nicely relevant to psychological aspects such as cognition or attention. There are two P300 sub-components that overlap at the scalp; P3b has a more centroparietal distribution and corresponds to the classical P300 recorded within an oddball paradigm after rare and task relevant events. P3a occurs after novel events independently of task relevance and is characterized by a more frontal distribution, a shorter latency and fast habituation. These sub-components reflect functionally different processes. P3a has been interpreted as an orienting response. P3b has been related to many different psychological constructs such as control information processing, the information content of the events, memory processes, the reorganization of an internal expectancy model. The parietal and temporal cortex are involved in the generation of the auditory P3b. Concerning P3a, the superior temporal plane, the association cortices, limbic structure and frontal as well as pre-frontal cortices appear to play a major role. With local recording the hippocampus shows the largest P300. It has been proved clinically that $\mathrm{P} 300$ potentials recorded at the scalp result from intracortical currents induced by post-synaptic potential. The P300 activity of the temporo-basal dipoles corresponds mainly to the classical P3b and that of the frontally oriented temporo-superior dipoles to P3a. An increase of P300 latency with age is found for 
the temporo-basal but not for the temporo-superior dipoles. Figure 1 illustrates some typical P3a and P3b waves from temporo-basal and temporo-superior dipoles [1].

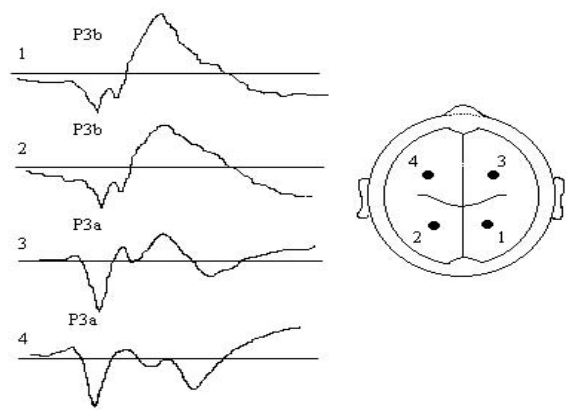

Fig. 1. Typical subcomponents of the P300 signals; a. P3a and b. P3b signals.

Attenuation of auditory and visual P300 signal can be a sign of schizophrenia. Detection and localization of the P300 source have been the objectives of psychiatry clinicians. Although in the healthy person P300 can be seen about 300 millisecond after applying the stimuli for the patients suffering from schizophrenia, the shape, amplitude, and even the position of the P300 may change. Furthermore, children of schizophrenic parents and other subjects with an enhanced risk of developing schizophrenia showed P300 abnormalities. Also small P300 amplitudes have been found in healthy sibling of schizophrenics. However, the reduction in the amplitude of P300 is also found in patients with dementia and with affective disorders. Furthermore the P300 is reduced only in a subgroup of schizophrenic patients. This indicates that the amplitude reduction of P300 is neither a sufficient nor a necessary marker of schizophrenic disorders. But it has been clinically observed that the patients with a P300 amplitude reduction are a sub-group with neuro-developmental disorders. Moreover, the amplitude reduction is more consistent in $\mathrm{P} 3 \mathrm{~b}$ subcomponents.

A reference signal can be modelled by averaging a number of (electroencephalogram (EEG) segments obtained after applying a periodic stimulus.

Blind separation of the EEG signals on the other hand, has been followed by a number of researchers [2] [3] [4]. Infomax algorithm [5] has been reported to be robust for separation of EEG signals. Some source separation problems such as signal detection and noise cancellation often expect to estimate a desired single source or a subset of sources from the mixtures. In such cases a separate objective function, as a constraint, has to be minimized (or maximized) in parallel with minimization of the original cost function. Exploitation of Lagrange multipliers [6] and nonlinear penalty functions [7] incorporate the constraint terms into the original cost functions thereby convert the constrained problems to unconstrained algorithms.

The BSS criterion (or equivalently ICA) for instantaneous mixtures such as EEGs, is formulated as follows. Denote the time varying observed signals by $\mathbf{x}=\left[x_{1}(t), x_{2}(t), \ldots, x_{n}(t)\right]^{T}$ where $\mathbf{x} \in R^{n}$ and the unknown independent sources $\mathbf{S}=\left[s_{1}(t), s_{2}(t), \ldots, s_{m}(t)\right]^{T}$ where $\mathbf{s} \in R^{m}$. 


$$
\mathbf{x}=A \mathbf{s}+\mathbf{v}
$$

and

$$
\mathbf{y}=W \mathbf{x}
$$

Here $\mathbf{v} \in R^{n}$ is assumed to be a white Gaussian noise vector, $A \in R^{m \times n}$ and $W \in R^{n \times m}$ are unknown constant mixing and unmixing matrices respectively, and (.) $\mathrm{T}$ is vector transpose. The mixture is assumed to be over-determined (valid for usual cases), i.e. $\mathrm{m}<\mathrm{n} . \mathbf{y}=\left[y_{1}(t), y_{2}(t), \ldots, y_{m}(t)\right]^{T}$, where $\mathbf{y} \in R^{m}$ is the output vector. The unconstrained separation matrix can be found by finding the global minima(or maxima) of a cost function $J_{M}(W)$, which provides a measure of independency of the estimated sources.

Incorporation of the constraint requires another cost function such as $J_{C}(W)$ to be minimized together with $J_{M}(W)$. The constraint term is then joined to the main objective function by using either a Lagrange multiplier or a set of penalty functions. Application of the Lagrange multiplier however, ignores nonlinearity of the system whereby the nonstationarity of the mixtures is not exploited. A general overall cost function is best defined as follows:

$$
J(\mathbf{W})=J_{M}(\mathbf{W})+k G\left(J_{C}(\mathbf{W})\right)
$$

where $G($.$) is the penalty function and k$ is the matrix of penalty coefficients. In the following sections a new constrained BSS method based on the original Infomax BSS system and incorporating of a reference signal as a constraint is introduced for detection and localization of the P300 sources within the brain from the EEG signals.

\section{Constrained Infomax Algorithm}

In an undetermined BSS system the estimated ICs do not necessarily represent the actual sources. This happens when EEGs are to be separated. In the development of this project we aim at separation of the scalp EEG mixtures in such away that the desired P300 signal is one of the estimated ICs. The Infomax BSS algorithm is based on minimization of the mutual information or maximization of the entropy. The Infomax cost function $J_{M}(\mathrm{w})$, can be found in the literature [5]. The unmixing matrix is recursively updated by finding a solution to the minimization of such unconstrained overall cost function i.e.

$$
\arg \min _{\mathbf{w}} J(\mathbf{w})=\arg \min _{\mathbf{w}}\left[J_{M}(\mathbf{w})+k G\left(J_{C}(\mathbf{w})\right)\right]
$$

where $J_{M}(\mathbf{w})$ is the main objective function of the Infomax BSS algorithm and

$$
J_{C}(\mathbf{W})=\|\mathbf{p}-\mathbf{y}\|^{2}=\|\mathbf{p}-\mathbf{w} \mathbf{x}\|^{2}
$$


where $\mathbf{p}$ is a matrix whose rows are the P300 reference signal. The reference signal is obtained by averaging several segments of the same electrode signal after a visual or auditory periodic stimulation. The update equation is generally denoted as

$$
\mathbf{w}(t+1)=\mathbf{w}(t)+\Delta \mathbf{w}(t)
$$

where by considering the extension to the NGA proposed by Amari [6] we have

$$
\begin{aligned}
\Delta \mathbf{w}(t) & =\mu \frac{\partial J(\mathbf{w})}{\partial \mathbf{w}} \mathbf{w}^{T} \mathbf{w} \\
& =\mu\left(\gamma \mathbf{I}+\left(1-\frac{2}{1+\exp (\mathbf{w x})}\right)(\mathbf{w} \mathbf{x})^{T}+2 \mathbf{q}\left(\mathbf{x}(\mathbf{w x}-\mathbf{p})^{T}\right)^{T} \mathbf{w}^{T}\right) \mathbf{w}
\end{aligned}
$$

Here $\mu$ is the learning rate, $\gamma$ is a constant, and $\mathbf{I}$ is a unitary matrix. $\mathbf{w}$ is initialised to $\mathbf{w}_{\text {init }}=\mathbf{I}$ and $\mu$ is calculated empirically via the following adaptive criterion:

$$
\mu(t)=\mu_{0}\left(\frac{\alpha}{\|\operatorname{Rx}\|_{F}^{2}}+\frac{\beta}{\zeta+\left\|\Delta J_{C}\right\|}\right)
$$

where $\mu_{0}, \alpha, \beta$, and $\zeta$ are constants adjusted for adaptation. In the above formulation $\mathbf{q}$ is updated iteratively based on the new $\mathrm{w}$ in the direction of minimizing the distance between the output ICs and the P300 reference signal i.e.

$$
\mathbf{q}=k \cdot \operatorname{diag}\left[(\mathbf{w} \mathbf{x}-\mathbf{p})(\mathbf{w} \mathbf{x}-\mathbf{p})^{T} \bigvee / p^{2}\right.
$$

where $k$ is the penalty parameter. In the above analysis we ignored the effect of noise, which is inherently contained in $\mathbf{x}$. However, incorporation of the constraint into the original Infomax update equation does not change the performance of the system in terms of noise effect.

\section{Localization Criterion}

Localization of the EEG sources has been investigated recently [3] [8] [9]. With some indeterminacy in the result we can approximate the location of the sources within the brain. Unlike the methods in [8] and [9], which consider the sources as magnetic dipoles, in our approach we simply consider them as the sources of isotropic propagating signals. Therefore the head (mixing media) model only mixes and attenuates the signals. Therefore based on Figure 2 we have

$$
\left\|\mathbf{f}_{k}-\mathbf{a}_{j}\right\|_{2}=d_{j}
$$

where $\mathbf{f}$ and $\mathbf{a}_{j}$ refer to the source and the electrode coordinates respectively, and $d_{j}$ are nonlinearly proportional to the inverse of the correlations between the estimated source $k$ and the electrode signals. $j=1,2,3$ represents the electrode involved in calculation of the correlation values, and $k=1,2, \ldots, M$, shows the source number. In this equation all the variables except $\mathbf{f}$ is known. Incorporating more than three mixtures does not affect the result whereas it makes the computation more intensive. The nonlinearity stated above comes from the fact that head is not a homogenous region. 
In a spherical model of the head we may consider three main layers; brain, skull, and scalp for which the thickness is known. The conductivity of the skull is about 100 times less than those of the brain and the scalp i.e. $\sigma_{\text {brain }}=\sigma_{\text {scalp }}=80 \sigma_{\text {skull }}$. In order to incorporate the non-homogeneity and ensure that there will be a solution to equation (10) within the brain region, these values have to be nonlinearly mapped and normalized such that all the estimated sources fall within the brain region. A more accurate method requires the information about non-homogeneity of the media between the sources and the electrodes including the brain (white and gray tissues), the skull and the scalp. Utilizing the described method, it has been shown that the P300 sources within the brain can be localized by a high degree of accuracy.

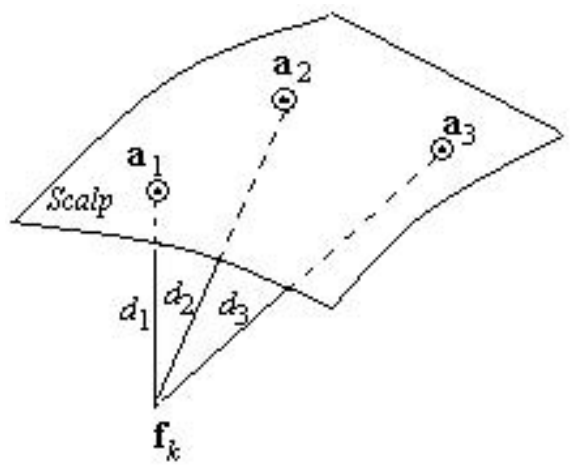

Fig. 2. Part of the scalp including three electrodes, and the location of the source to be identified (assuming the head is homogenous).

\section{Experimental Results}

In this part the proposed CBSS algorithm is applied to the simulated as well as real EEG data from both a healthy person and a schizophrenia patient. The sampling frequency is 200 sample/sec. The data is pre-whitened and $\mathbf{w}$, the separation matrix is initialized to $\mathbf{I}$. The P300 reference signal for natural mixtures is achieved by averaging a number of segments of each electrode signal. This signal is then used as a reference for updating the unmixing matrix.

The algorithm maximizes the P300 component as an output of the unmixing matrix. In all the cases the convergence of the algorithm is very fast. In the first experiment only three mixtures including the P300 have been modelled and used. Figure 3 compares the results of separation using traditional Infomax BSS algorithm and those of the proposed constrained technique. Figure 4 shows the convergence of the system with respect to that of the well-known NGA-based joint diagonalization criterion. It is very obvious that the new system converges much faster than the conventional NGA. In the next two experiments the reference P300 signal is estimated from the mixtures by taking an average of a number of segments for each electrode. To have a better reference signal a periodic visual (or audio) stimulation is necessary. The period of 

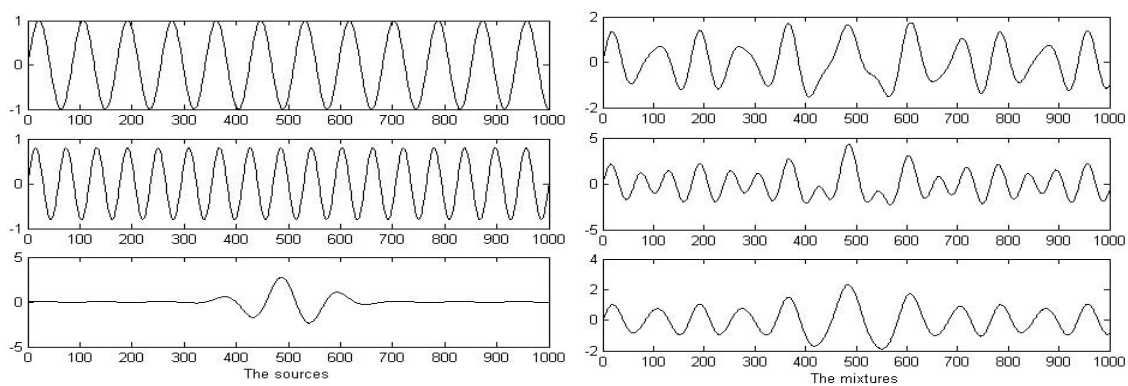

(a)

(b)
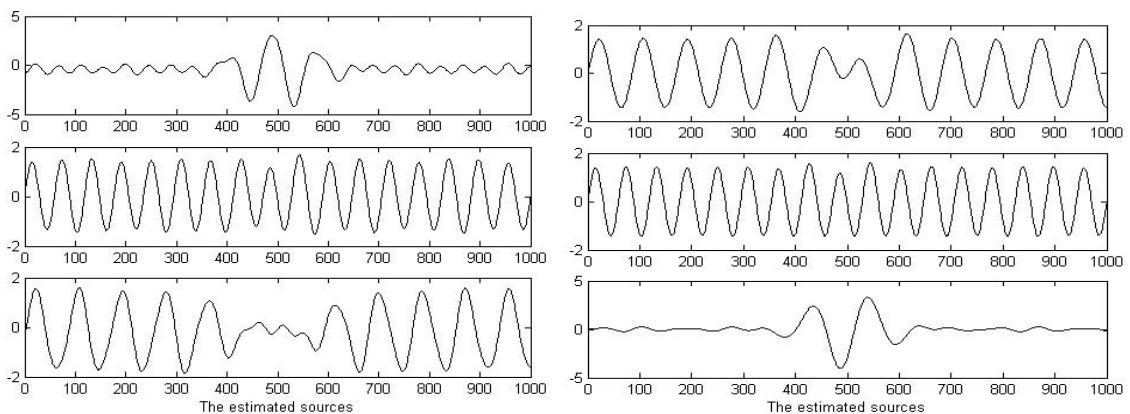

(c)

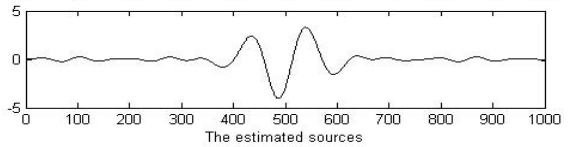

(d)

Fig. 3. The results of separation of simulated P300 signal; (a) the original sources, (b) the mixtures, (c) the estimated sources using the traditional and (d) the estimated sources using the constrained Infomax algorithms.

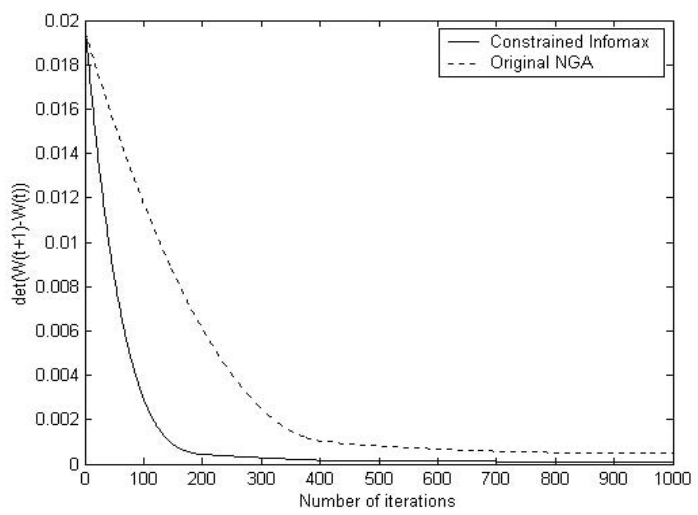

Fig. 4. The convergence comparison between the CBSS and the traditional NGA.

the stimulation is ten seconds and it lasts for one second. We avoid any eye blinking or other artifacts. Figure 5 illustrates the results from separation of the P300 signal for a schizophrenia patient. In this case the P300 signal is not clear in the EEG signals. 


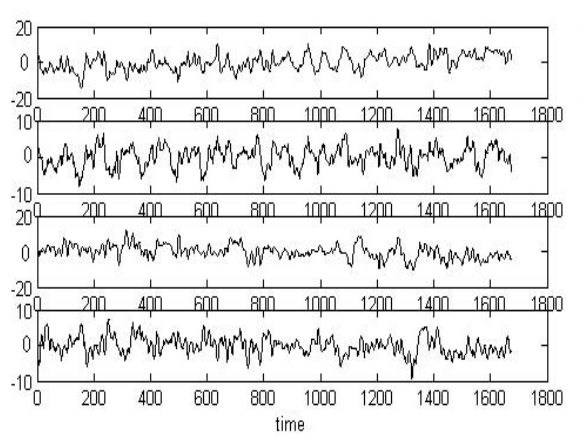

(a)

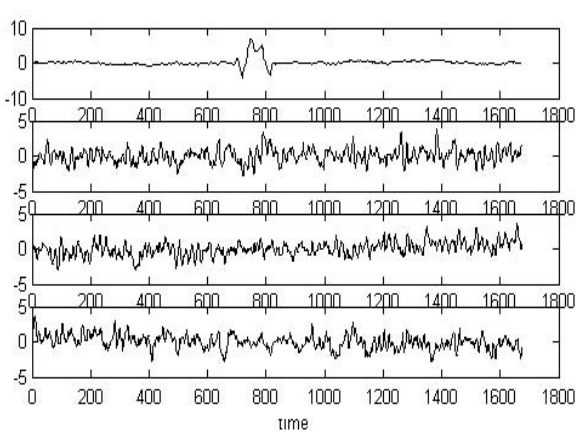

(b)

Fig. 5. The results of separation of a real P300 signal in a schizophrenia patient, using the constrained Infomax algorithm; (a) the EEGs and (b) the separated signals using CBSS, the top signal is the separated P300 (only 4 out of 10 signals have been displayed here).

After separation, the estimated highlighted component is localized. In order to guarantee a solution to equation (10) the following steps were followed: (a) The correlation values are inversed and normalized into between 0 and 1, (b) A nonlinear $\mu$ law function, with $100>\mu>25$, was used for transformation of the correlation values, (c) The transformed values were scaled into between $12 \mathrm{~mm}$ and the radius of the head, as $d_{j}$, and (d) The solution to the following least square problem was obtained [10]:

$$
\min S\left(\mathbf{f}_{k}\right), \quad \mathbf{f}_{k} \in R^{n}, \text { where } S\left(\mathbf{f}_{k}\right)=\sum_{j=1}^{3}\left\{\left\|\mathbf{f}_{k}-\mathbf{a}_{j}\right\|_{2}-d_{j}\right\}^{2}
$$

Following the above steps for a head phantom, in more than $95 \%$ of the cases an exact localization of the sources has been achieved. Both P3a and P3b signals are localized around the temporo-superior dipoles and temporo-basal dipoles respectively. Based on the information achieved in this project an informative display platform has been provided which greatly assist the clinicians in diagnosis of neurological disorders.

\section{Conlusions}

An effective and robust CBSS method has been developed and used for separation and localization of the P300 sources in healthy individuals and the schizophrenic patients. The algorithm is an extension of original Infomax algorithm, based on minimization of mutual information, for which a reference P300 signal is used as a constraint. The constrained problem is then converted to an unconstrained problem by means of nonlinear penalty functions weighted by the penalty terms. The algorithm has been examined on both simulated and natural EEG signals of both healthy and schizophrenic patients. Both auditory and visual P300 can be separated and well localized. The reference signal forces the unmixing matrix to separate the sources of 
P300 resulted from the corresponding stimulations. The results are compared with those of the traditional Infomax algorithm and the traditional NGA method in terms of the convergence speed. The method is an effective tool in investigation of the schizophrenia disease (as well as some other neurological disorders such as Alzheimer's) in neurophysiology and psychiatry departments.

\section{References}

1. E. Niedermeyer and F. L. Da Silva, Electroencephalography; basic principles, clinical applications, and related fields, Ed. 4, LW\&W, (1999).

2. R. N. Vigario, "Extraction of ocular artefacts from EEG using independent component analysis," Electroencephalography and Clinical Neurophysiology, 103, pp. 395-404, (1997).

3. A. Cichocki et al., "Neural networks for blind separation with unknown number of sources," Neurocomputing, 24(1-3): pp. 55-93, February (1999).

4. S. Makeig, et al. "Independent component analysis of electroencephalographic data," Advances in neural information processing systems 8, pp. 145-151 MIT Press, Cambridge, MA, (1996).

5. J. F. Cardoso, "Infomax and maximum likelihood for blind source separation,” IEEE Signal Processing Letter, 4, pp. 109-111, April (1997).

6. A. Cichocki and S. I. Amari, Adaptive blind signal and image processing, J. Wiley, (2002).

7. W. Wang, S. Sanei, and J. A. Chambers, "Penalty function based joint diagonalization approach for convolutive blind separation of nonstationary sources," to be published in IEEE Transactions on Signal Processing.

8. J. C. Mosher \& R. M. Leahy, "Source localization using recursively applied and projected (RAP) MUSIC," IEEE Trans. on SP, 47(2), pp. 332-340, Feb. (1999).

9. J. C. Mosher \& R. M. Leahy, and P. S. Lewis, "EEG and MEG: Forward solutions for inverse methods," IEEE Trans. on Biomedical Engineering, 46(3), pp. 245-259, March (1999).

10. I. D. Coope, "Reliable computation of the points of intersection of $n$ spheres in $R^{n}$," ANZIAM J., 42(E), pp. C461-C477, (2000). 